\title{
Crystal Nucleation and Growth of Natural Rubber Purified by Deproteinization and Trans-esterification
}

\author{
Seiichi Kawahara, ${ }^{\dagger}$ Keiko TAKano, Jintana YunyongwattanaKorn, ${ }^{*}$ Yoshinobu Isono, \\ Masamichi HiKosAKa, ${ }^{* *}$ Jitladda T. SAKDAPIPANICH, ${ }^{*}$ and Yasuyuki TANAKA*** \\ Department of Chemistry, Faculty of Engineering, Nagaoka University of Technology, Nagaoka 940-2188, Japan \\ *Department of Chemistry, Faculty of Science, Mahidol University, Rama VI, Bangkok 10400, Thailand \\ ${ }^{* *}$ Soft Materials Physics Group, Faculty of Integrated Arts and Sciences, Hiroshima University, \\ Kagamiyama, Higashi-Hiroshima 739-8521, Japan \\ ***Institute of Science and Technology for Research and Development, Mahidol University, \\ Salaya Campus, Nakornprathom 73170, Thailand
}

(Received May 23, 2003; Accepted January 13, 2004; Published May 15, 2004)

\begin{abstract}
The crystallization of highly purified natural rubber (NR) was investigated by polarized light microscopy. NR was deproteinized with proteolytic enzyme in the presence of surfactant and further purified by acetone-extraction followed by transesterification to remove all mixed and linked fatty acids present in NR. The rubber was fractionated and characterized by size exclusion chromatography, ${ }^{1} \mathrm{H}$ and ${ }^{13} \mathrm{CNMR}$ spectroscopy. Fractionated rubbers were crystallized at $252-258 \mathrm{~K}$, in which spherulite was observed. The estimated nucleation and growth rates depended on supercooling. Surface free energies were determined for highly purified NR. Nucleating effect of fatty acids on the crystallization was proved by change in the surface free energy after removing and/or adding fatty acids.
\end{abstract}

[DOI 10.1295/polymj.36.361]

KEY WORDS Natural Rubber / Deproteinization / Transesterification / Polarized Light Microscopy / Crystal Nucleation / Growth / Surface Free Energy /

Natural rubber (NR), obtained from Hevea brasiliensis, crystallizes below ambient temperature, ${ }^{1}$ which may depend on the long sequence of cis-1,4 isoprene repeating units, ${ }^{2}$ branching structure, and non-rubber components such as proteins and phospholipids present in NR. ${ }^{3}$ The rapid crystallization of NR, compared to synthetic analogous, is a long-standing puzzles, due to little information on the effects of branching structure and non-rubber components on crystallization behavior. Recently, we proposed the fundamental structure of NR, that is, $\omega$-terminal, two trans-1,4 isoprene units, about 1,000-5,000 cis1,4 isoprene units and $\alpha$-terminal linking up with two or three fatty acids per rubber chain, aligned in that order. ${ }^{4}$ Structural studies using NMR spectroscopy suggest that the $\omega$-terminal contains a functional group composed of modified dimethylallyl group, which forms branch points through hydrogen bonding between proteins, while the $\alpha$-terminal consists of a phospholipid group, which forms chemical branching points. These hypothetical branching points, forming three-dimensional network structure, are shown in Figure 1. These branching points may be formed during preservation of NR as ammoniated latex, since gel content of NR increases from less than $1 \mathrm{w} / \mathrm{w} \%$ of fresh latex to more than $50 \mathrm{w} / \mathrm{w} \%$ in high ammonia latex stored longer than 8 weeks. ${ }^{5}$
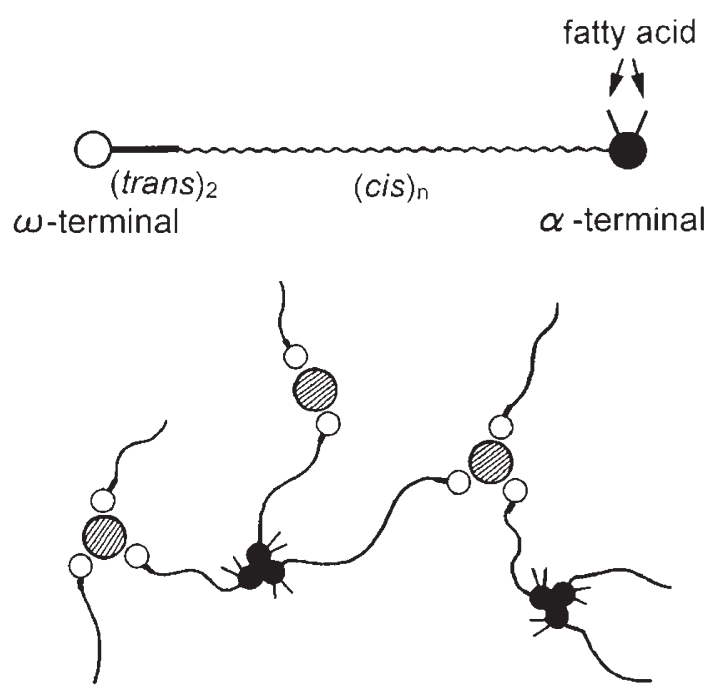

Figure 1. Presumed structure of branching points in natural rubber.

Crystal growth of NR has been studied by transmission electron microscopy (TEM) of stained thin films, prepared from fractionated rubbers having various molecular weights. ${ }^{6}$ For these fractionated rubbers, molecular weight was found to be a function of the number of branching points. ${ }^{7}$ Thus, growth rates reported by Phillips should include the effects of not on-

${ }^{\dagger}$ To whom correspondence should be addressed (Tel: 81-258-47-9301, Fax: 81-258-47-9300, E-mail: kawahara@chem.nagaokaut.ac.jp). 
ly the molecular weight and supercooling but also the number of branching points. In view of the effects of branching points in NR on the crystallization behavior, it is quite important to prepare purified NR composed of linear rubber chains.

To investigate the effects of molecular weight and supercooling on the crystallization of NR, we prepared NR composed of linear chains. In previous studies, ${ }^{8,9}$ we removed almost all proteins in NR using a proteolytic enzyme in conjunction with a surfactant. Phospholipids in NR were decomposed by a transesterification with sodium methoxide. ${ }^{8}$ Based on the proposed backbone structure, the rubber should be a linear molecule. ${ }^{10}$ In the present study, we investigate the crystallization of the highly purified NR. Crystal nucleation and growth for fractionated rubbers and highly purified rubber mixed with stearic acid are observed by polarized light microscopy.

\section{EXPERIMENTAL}

The natural rubber (NR) latex used in this study was fresh field latex obtained from Malaysia. The latex was immediately subjected to enzymatic deproteinization through incubation with $0.04 \mathrm{w} / \mathrm{v} \%$ proteolytic enzyme (Novo Nordisk Alcalase 2.0T) and $1 \mathrm{w} / \mathrm{v} \%$ sodium dodecyl sulfate (SDS) for $12 \mathrm{~h}$ at $311 \mathrm{~K}$ followed by centrifugation. The cream fraction was re-dispersed in $1 \mathrm{w} / \mathrm{v} \%$ SDS to make $30 \mathrm{w} / \mathrm{w} \%$ dry rubber content (DRC) latex and was washed twice by centrifugation to prepare deproteinized natural rubber latex. The rubber was recovered by centrifugation followed by coagulation with methanol and dried under reduced pressure at ambient temperature until a definite weight was achieved.

The rubber was extracted with acetone in a Soxhlet for $24 \mathrm{~h}$ under nitrogen atmosphere. Transesterification of the acetone-extracted rubber was carried out in toluene with fresh sodium methoxide under nitrogen atmosphere. The transesterified natural rubber (NRTE) was fractionated using toluene and methanol into five fractions: fraction $1(\mathrm{Fr} 1)$, fraction $2(\mathrm{Fr} 2)$, fraction $3(\mathrm{Fr} 3)$, fraction $4(\mathrm{Fr} 4)$, and fraction 5 $(\mathrm{Fr} 5)$. The fractionated rubbers were dried under reduced pressure at room temperature.

Measurements of molecular weight and molecular weight distribution of the rubbers were made with a TOSOH GPC, consisting of a TOSOH CCPD pump, RI-8012 differential refractometer and UV-8011 UV detector. A column with an exclusion limit of about $4 \times 10^{7}$ was used. Measurement was made at $303 \mathrm{~K}$ and flow rate of the mobile phase, THF, was 0.5 $\mathrm{mL} / \mathrm{min}$.

${ }^{1}$ HNMR measurement was carried out using a JEOL EX-400 NMR spectrometer operating at
399.65 MHz. The polymer was dissolved in benzene- $d_{6}$. The measurement was carried out at $323 \mathrm{~K}$ at pulse repetition times of $7 \mathrm{~s}$.

Crystallization of the fractionated rubbers was observed by polarized light microscopy (PLM), using a Nikon Eclipse E600 POL. The sample, sandwiched between two cover glasses, was placed on a cooling stage (Linkam LK 600PM) whose temperature control was maintained within $0.1 \mathrm{~K}$. The sample was heated at $323 \mathrm{~K}$ to melt the preexisting crystals before crystallization. The melted rubbers were crystallized at $252-258 \mathrm{~K}$. The number of spherulites near the center of the sample was counted by PLM, while the size of the spherulites was measured as the longest ellipsoidal diameter. Melting temperature was determined at a heating rate of $1 \mathrm{~K} / \mathrm{min}$ after crystallization. Temperature was calibrated using standard materials, indium and linoleic acid. To observe spherulites, observation was limited to the earlier stage of crystallization.

Melting behavior of the fractionated rubbers was examined with a DSC 220 by Seiko Instruments Inc., calibrated with standard indium and linoleic acid before measurement. The polymer packed into an aluminum pan was heated at $353 \mathrm{~K}$ to melt preexisting crystal before crystallization. The melted polymer was cooled to the crystallization temperature as soon as possible with liquid nitrogen and crystallized. After crystallization, DSC measurement was made from $153 \mathrm{~K}$ to $373 \mathrm{~K}$ at a heating rate of $10 \mathrm{~K} / \mathrm{min}$. Melting temperature was determined as a peak top of the melting endotherm of the DSC thermogram.

\section{RESULTS AND DISCUSSION}

\section{Characterization}

Chain-end groups of the lowest molecular weight fraction were analyzed by ${ }^{1} \mathrm{HNMR}$ spectroscopy, as shown in Figure 2. The major signals characteristic of NR appearing at 1.76, 2.10 and $5.13 \mathrm{ppm}$ were assigned to methyl, methylene and unsaturated methyne protons of cis-1,4-isoprene repeating units, respectively. Small signals were observed at $1.66-1.76 \mathrm{ppm}$. These were assigned based on signals observed in model compounds, well characterized in previous studies, ${ }^{7,10,11}$ as shown in Figure 2. Two signals at 1.66 and $1.75 \mathrm{ppm}$ were assigned to methyl protons of isoprene units in the trans-trans-cis triad sequence at the $\omega$-terminal ${ }^{11}$ and $c i s-c i s-\alpha$ present at the $\alpha$-terminal, ${ }^{7,10}$ respectively. The signal of the methyl proton due to $\omega$-trans-trans sequence, expected to appear at $1.68 \mathrm{ppm}$, was not observed in the spectrum. This may be explained as due to chemical modification of the $\omega$-terminal, inferred from plausible structures for modified dimethylallyl-groups, i.e., methylenecarbon atoms in dimethylallyl groups linking up with 


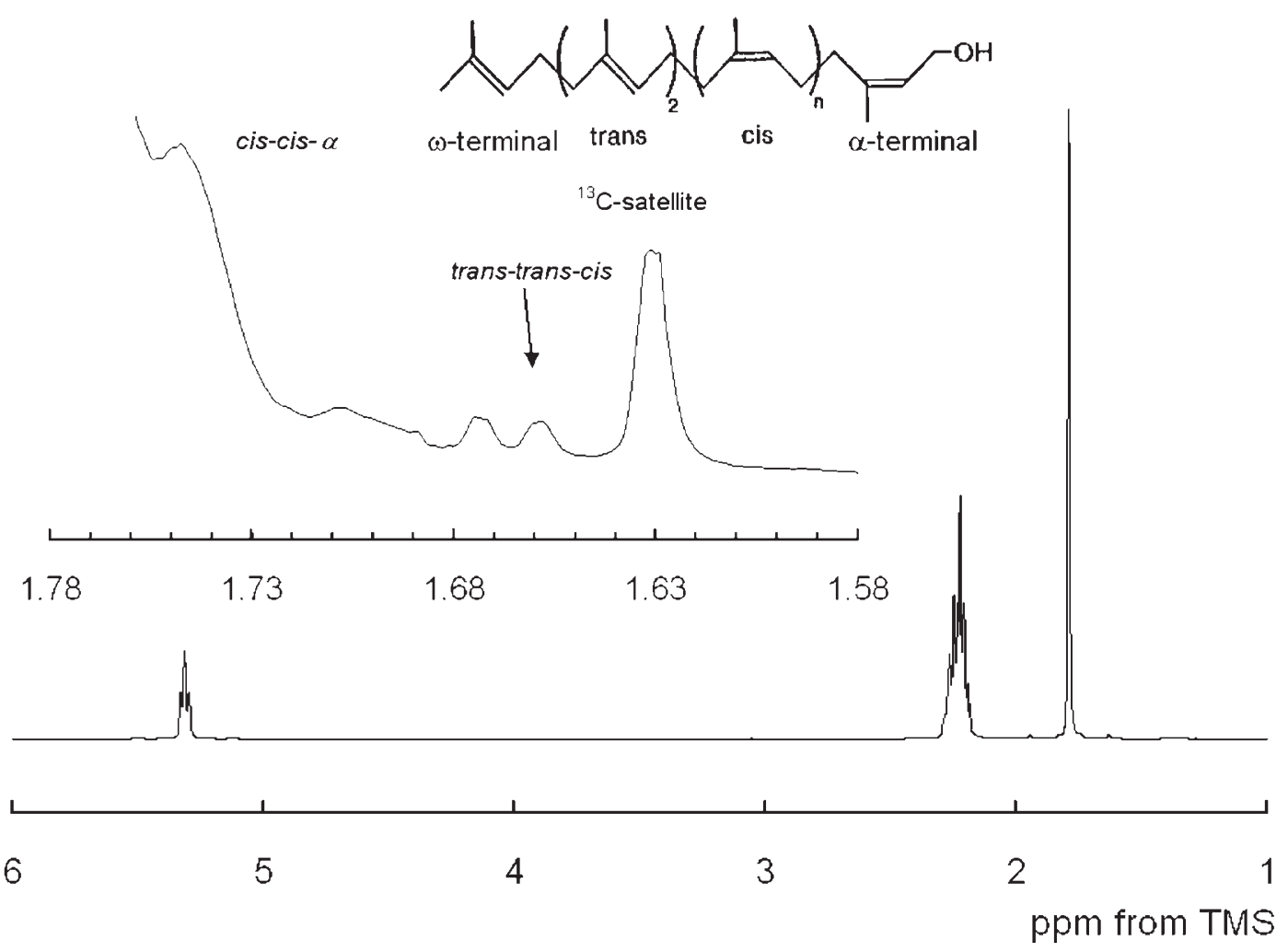

Figure 2. ${ }^{1}$ H NMR spectrum of Fr5.

an unidentified functional group. As an index of residual protein in deproteinized NR, we measured nitrogen content, $N \%$, which was less than $0.02 \%$, suggesting the decomposition of almost all proteins, based on previous work. ${ }^{8}$

Size exclusion chromatogram (SEC) for fractionated rubbers is shown in Figure 3. A unimodal, symmetrical peak was observed for all fractions, as reported previously. ${ }^{10}$ The number average molecular weight, $M_{\mathrm{n}}$, weight average molecular weight, $M_{\mathrm{w}}$, and polydispersity, $M_{\mathrm{w}} / M_{\mathrm{n}}$, of fractionated samples were estimated from intensity and elution volume of the peak using a calibration curve drawn with standard polystyrene samples; values of $M_{\mathrm{w}}$ and $M_{\mathrm{w}} / M_{\mathrm{n}}$ are tabulated in Table I. $M_{\mathrm{w}}$ of the fractionated rubber ranged from $6.76 \times 10^{4}$ to $1.11 \times 10^{6}$.

$M_{\mathrm{n}}$ determined by SEC for the lowest molecular weight fraction was converted to that of polyisoprene (PI) using an empirical expression, as reported by Subramaniam: ${ }^{12}$

$$
\log M_{\mathrm{n}, \mathrm{PI}}=0.185+0.950 \log M_{\mathrm{n}}
$$

Estimated $M_{\mathrm{n} \text {,PI }}$ shown in Table I is quite similar to $M_{\mathrm{n}, \mathrm{NMR}}$ estimated from the intensity ratio of methyl proton signals of cis-cis-cis to trans-trans-cis sequence, assuming that a linear chain contains two trans-1,4 units and long sequence of cis-1,4 units. ${ }^{7,11}$ This supports that purified NR is composed of linear chains.

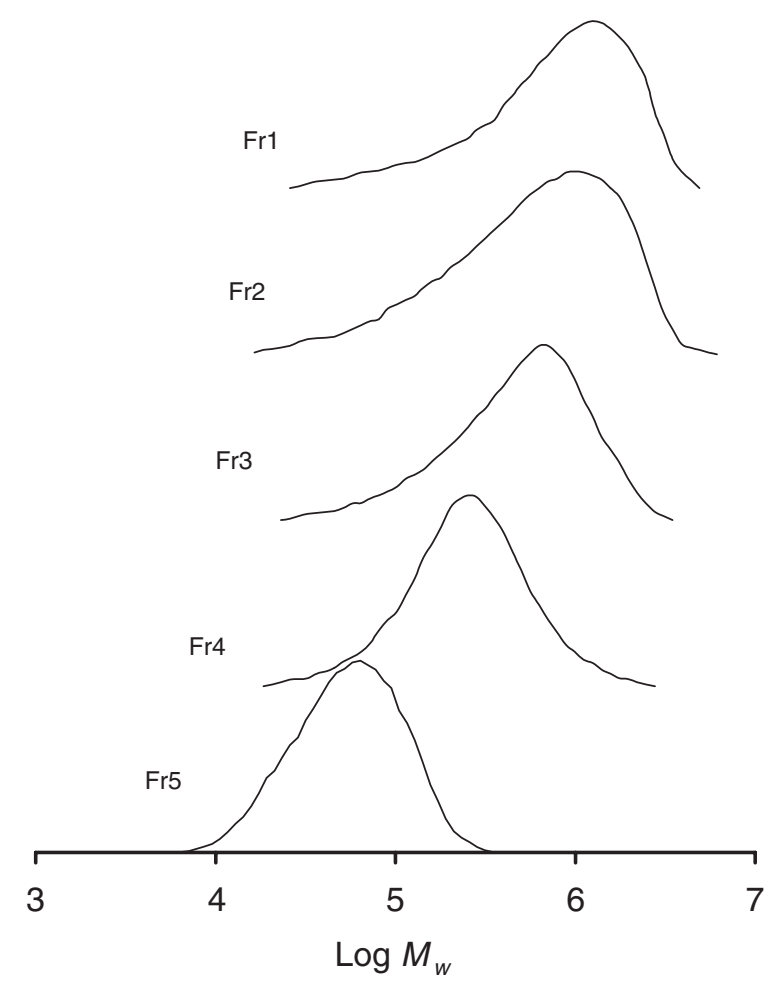

Figure 3. Size exclusion chromatogram for fractionated rubbers.

\section{Crystallization of the Fractionated Rubbers}

Crystallization of fractionated rubbers was examined by polarized light microscopy (PLM). Typical 
Table I. $M_{\mathrm{n}}, M_{\mathrm{w}} / M_{\mathrm{n}}$, and $N \%$ for fractionated rubbers

\begin{tabular}{cccccc}
\hline Specimen & $M_{\mathrm{w}} / 10^{4}$ & $M_{\mathrm{w}} / M_{\mathrm{n}}$ & $M_{\mathrm{n}, \mathrm{PI}} / 10^{4}$ & $M_{\mathrm{n}, \mathrm{NMR}} / 10^{4}$ & $N \%$ \\
\hline Fr1 & 111 & 2.5 & & & \\
Fr2 & 89.9 & 3.1 & & & \\
Fr3 & 65.6 & 2.1 & & & \\
Fr4 & 33.6 & 1.8 & & & \\
Fr5 & 6.76 & 1.6 & 6.09 & 5.5 & 0.019 \\
\hline
\end{tabular}

micrographs for Fr4 are shown in Figure 4. Small spherulites appeared after induction, and size increased with crystallization time, $t$. The spherulites showed a Maltase-cross observed by PLM, suggesting that radial growth of the spherulites occurs during crystallization. This is consistent with the spherulites observed by Andrews using TEM. ${ }^{13}$ Typical changes in the number of spherulites per unit volume, $v$, and size, $a$, for Fr4 vs. time are shown in Figures 5 and 6, respectively. $v$ increased gradually and then linearly in the steady nucleation period, while $a$ increased linearly, as shown in Figure 6. Thus, we estimated the nucleation rate, $I$, as the slope in the plot of $v v s$. time and growth rate, $V$, as the slope in the plot of $a v s$. time.

Table II lists data of melting temperature, $T_{\mathrm{m}}$, for Fr1, determined by PLM and DSC. At any crystallization temperature, $T_{\mathrm{m}}$ determined by PLM was similar to that determined by DSC. Thus, $T_{\mathrm{m}}{ }^{0}$ was estimated from $T_{\mathrm{m}}$ determined by PLM. $T_{\mathrm{m}}$ was plotted against reciprocal lamellar thickness, $l$, where $l$ is a function of crystallization temperature, $T_{\mathrm{c}}$, according to Dalal ${ }^{15}$ as follows;

$$
l=9.93+0.228 T_{\mathrm{c}}+0.297 \times 10^{-2} T_{\mathrm{c}}{ }^{2}
$$

Lamellar thickness and $T_{\mathrm{m}}$ of purified natural rubber were similar to the corresponding values for untreated natural rubber. ${ }^{14}$ As shown in Figure 7, $T_{\mathrm{m}}$ was independent of molecular weight and proportional inversely to $l$, as,

$$
T_{\mathrm{m}}=T_{\mathrm{m}}{ }^{0}\left(1-\frac{2 \sigma_{\mathrm{e}}}{\Delta h_{\mathrm{f}} l}\right)
$$

where $\Delta h_{\mathrm{f}}$ is heat of fusion per unit volume of a crystal and $\sigma_{\mathrm{e}}$ fold surface free energy. In terms of Eq 3, we made an extrapolation of the linear line of $T_{\mathrm{m}}$ vs. $1 / l$ to $1 / l=0$. Estimated $T_{\mathrm{m}}{ }^{0}$ was $309 \mathrm{~K}$, similar to $T_{\mathrm{m}}{ }^{0}$, i.e., $308.7 \mathrm{~K}$ reported by Dalal. ${ }^{15}$ Using $\Delta h_{\mathrm{f}}=6.4 \times 10^{7} \mathrm{~J} \mathrm{~m}^{-3}$ reported by Kim and Mander$\mathrm{kern}^{16}$ and $T_{\mathrm{m}}{ }^{0}=309 \mathrm{~K}, \sigma_{\mathrm{e}}$ was estimated from the slope of the line to be $0.024 \mathrm{~J} \mathrm{~m}^{-2}$.

Figure 8 shows plots of logarithmic $V$ vs. $1 / T \Delta T$, for Fr1, Fr2, Fr3 and Fr4 reported in our previous paper, ${ }^{17}$ where $T$ is absolute temperature, $\Delta T$ supercooling, or $\Delta T=T_{\mathrm{m}}{ }^{0}-T$. The logarithmic $V$ was inversely proportional to $T \Delta T$, and decreased with

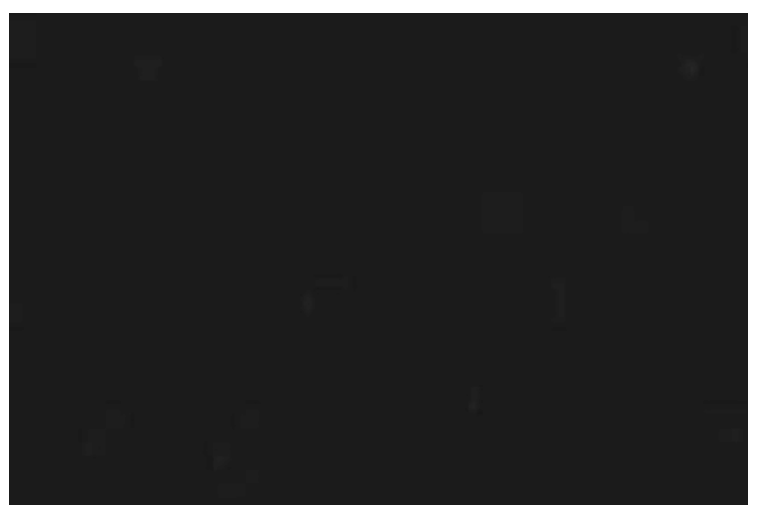

\section{Omin}

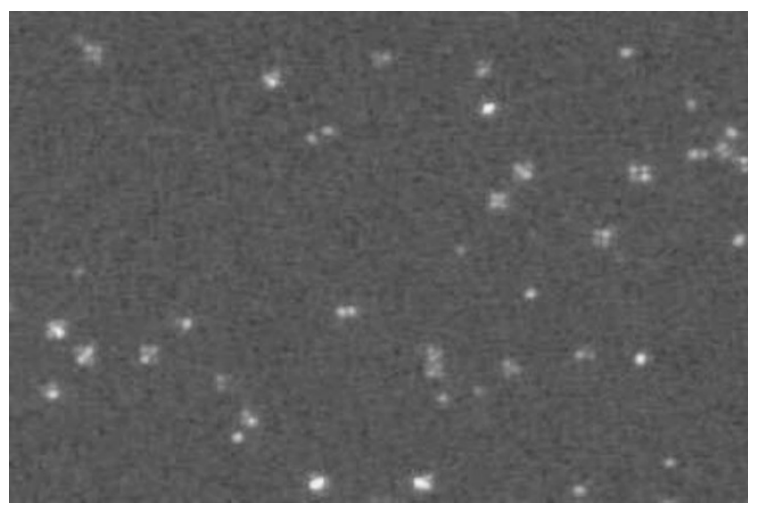

$150 \mathrm{~min}$

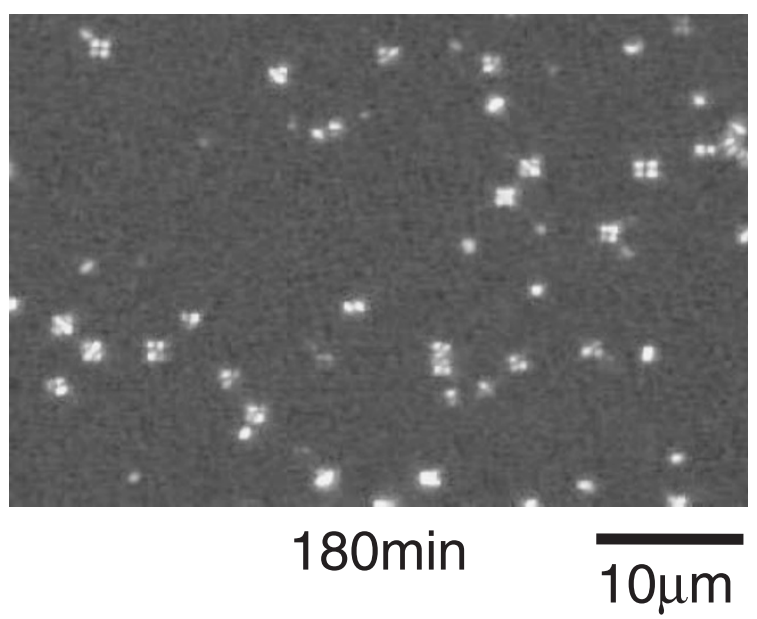

Figure 4. Polarized light micrographs for Fr4 crystallized at $252 \mathrm{~K}$.

molecular weight. $V$ is expressed as,

$$
\begin{aligned}
& \ln V=\ln V_{0}-\left(K_{\mathrm{V}} / T \Delta T\right) \\
& K_{\mathrm{V}}=4 \sigma \sigma_{\mathrm{e}} T_{\mathrm{m}}{ }^{0} / 3 k \Delta h_{\mathrm{f}}
\end{aligned}
$$

where $\sigma$ is lateral surface free energy, $k$ Boltzmann constant, 3 due to three dimensional growth for spherulite. Equations 4 and 5 suggest that the slope of the line in plots of $\ln V v s .1 / T \Delta T$ is a surface free energy term. Since the slope of the line was the same for all 


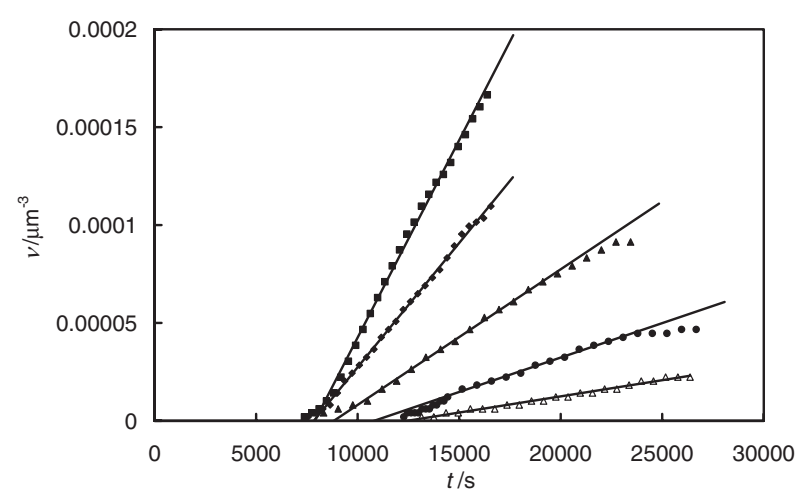

Figure 5. Number of crystals per unit volume, v, for Fr4 at crystallization temperature of $(\square) 250 \mathrm{~K},(\diamond) 252 \mathrm{~K},(\boldsymbol{\Delta}) 254 \mathrm{~K}$, (๑) $256 \mathrm{~K}$, and $(\triangle) 258 \mathrm{~K}$.

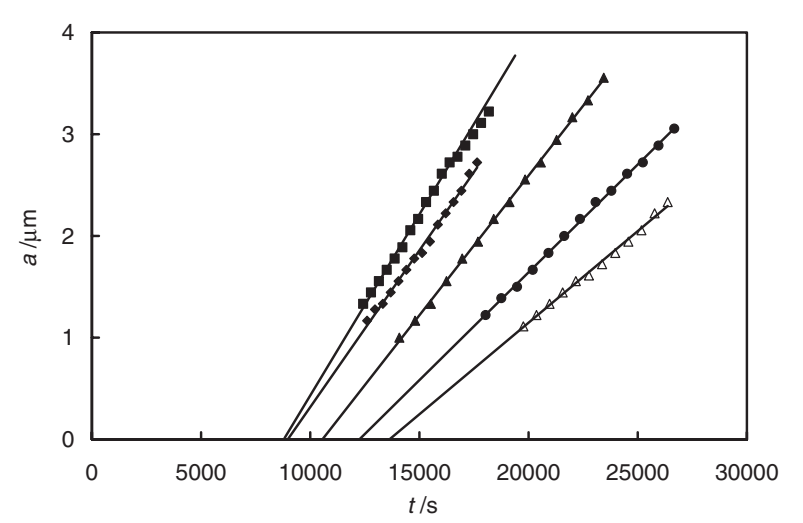

Figure 6. Size of crystal, $a$, for Fr4 at crystallization temperatures of $(\boldsymbol{\square}) 250 \mathrm{~K},(\bullet) 252 \mathrm{~K},(\boldsymbol{\Delta}) 254 \mathrm{~K},(\bullet) 256 \mathrm{~K}$, and $(\triangle)$ $258 \mathrm{~K}$.

Table II. Melting temperature, $T_{\mathrm{m}}$, for Fr1, determined by PLM and DSC

\begin{tabular}{ccc}
\hline$T_{\mathrm{c}}{ }^{\mathrm{a}}$ & $\begin{array}{c}T_{\mathrm{m}, \mathrm{PLM}} \\
(\mathrm{K})\end{array}$ & $\begin{array}{c}T_{\mathrm{m}, \mathrm{DSC}} \\
(\mathrm{K})\end{array}$ \\
\hline 260 & 278.3 & \\
258 & 277.5 & 277.6 \\
256 & 276.5 & 276.7 \\
254 & 275.3 & 275.6 \\
252 & 274.0 & 274.7 \\
250 & & 273.4 \\
248 & & 272.3 \\
\hline
\end{tabular}

${ }^{\mathrm{a}}$ Crystallization temperature of rubber.

fractions, as shown in Figure 8, we estimated $\sigma$ to be $0.013 \mathrm{~J} \mathrm{~m}^{-2}$. This is in good agreement with the reported value ${ }^{19}$ of $0.014 \mathrm{~J} \mathrm{~m}^{-2}$. Phillips ${ }^{6}$ reported that $\sigma_{\mathrm{e}}$ depended on molecular weight but $\sigma$ did not. However, in the present study, we found that not only $\sigma_{\mathrm{e}}$ but also $\sigma$ were independent of molecular weight, as in the case of polyethylene. Since, in the present study, we removed almost all proteins and phospholipids in natural rubber, most branching points should

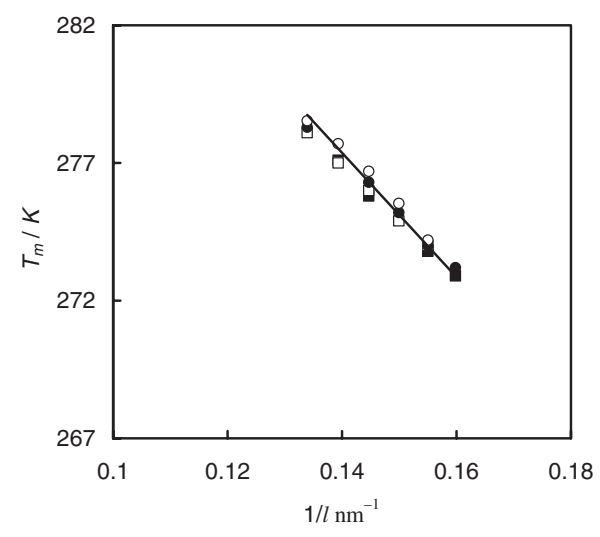

Figure 7. Plots of $T_{\mathrm{m}} v$ s. $1 / l$ for $(\bigcirc) \mathrm{Fr} 1,(\bullet) \mathrm{Fr} 2$, ( $\left.\square\right) \mathrm{Fr} 3$, and (口) Fr4

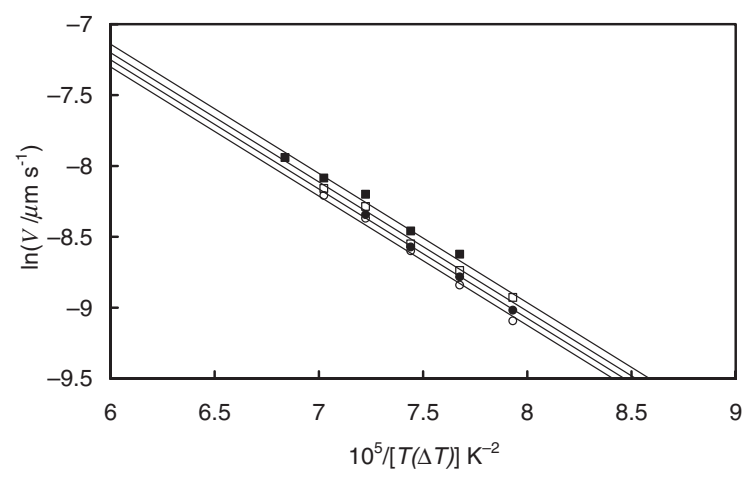

Figure 8. Semi-logarithmic plots of growth rate vs. $1 / T \Delta T$ for $(\bigcirc) \operatorname{Fr} 1,(\bullet) F r 2,(\square) \mathrm{Fr} 3$, and $(\square)$ Fr4, quoted from Ref. 17 in a rearranged style.

decompose. Consequently, it may be concluded that $\sigma_{\mathrm{e}}$ and $\sigma$ are independent of molecular weight. In Figure 8, the linear line moves downward as molecular weight increases. $\ln V_{0}$ in Eq 4 may thus be a function of molecular weight. This is due to the nature of $V_{0}$ that reflects topological diffusion of crystallization. $^{20}$

A semi-logarithmic plot of $I$ vs. $1 / T \Delta T^{2}$, for Fr1, $\mathrm{Fr} 2, \mathrm{Fr} 3$ and Fr4 is shown in Figure 9. Logarithmic $I$ was inversely proportional to $T \Delta T^{2}$ and slope depended on molecular weight. Thus, we applied the following expression ${ }^{18}$ to the temperature dependence of logarithmic $I$, since there was a possibility of heterogeneous nucleation.

$$
\begin{aligned}
& \ln I=\ln I_{0}-\left(K_{\mathrm{I}} / T \Delta T^{2}\right) \\
& K_{\mathrm{I}}=16 \sigma \sigma_{\mathrm{e}}(\Delta \sigma)\left(T_{\mathrm{m}}{ }^{0}\right)^{2} / k \Delta h_{\mathrm{f}}{ }^{2}
\end{aligned}
$$

where $\Delta \sigma=\sigma+\sigma_{0 N}-\sigma_{N}, \sigma_{0 N}$ is interfacial free energy between rubber crystals and substrate, and $\sigma_{N}$ interfacial free energy between rubber melt and substrate. $\Delta \sigma$ must be less than $2 \sigma$ and, practically, is $2 \sigma$ for homogeneous nucleation. Estimated $\sigma$ and $\Delta \sigma$ are tabulated in Table III. $\Delta \sigma$ was small, demonstrating heterogeneous nucleation. Thus, as a nucleat- 


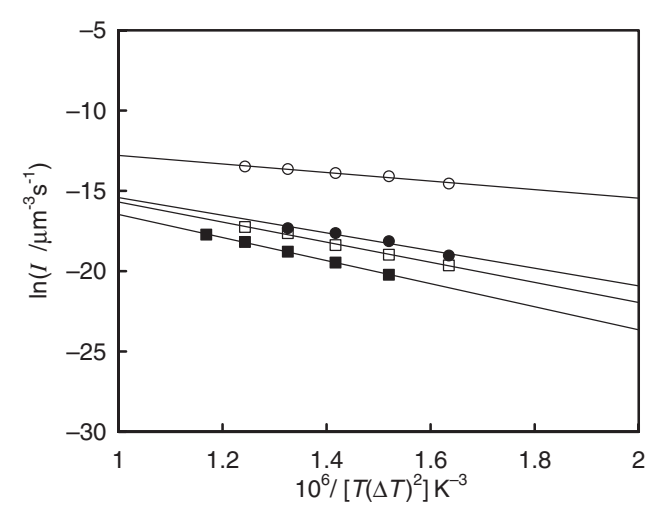

Figure 9. Semi-logarithmic plots of nucleation rate $v s$.

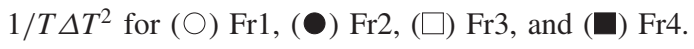

Table III. Observed $\sigma$ and $\Delta \sigma$ for fractionated natural rubber

\begin{tabular}{cccc}
\hline Specimen & $\begin{array}{c}\sigma \\
\left(10^{-2} \mathrm{~J} \mathrm{~m}^{-2}\right)\end{array}$ & $\begin{array}{c}\Delta \sigma \\
\left(10^{-4} \mathrm{~J} \mathrm{~m}^{-2}\right)\end{array}$ & $\begin{array}{c}\Delta \sigma / \sigma \\
\left(10^{-2}\right)\end{array}$ \\
\hline $\mathrm{NR}^{\mathrm{a}}$ & 1.4 & & \\
Fr1 & 1.3 & 3.1 & 2.4 \\
Fr2 & 1.3 & 6.5 & 5.0 \\
Fr3 & 1.3 & 7.4 & 5.7 \\
Fr4 & 1.3 & 8.5 & 6.6 \\
\hline
\end{tabular}

${ }^{\mathrm{a}} \sigma$ reported by Phillips. ${ }^{6}$

ing index, $\Delta \sigma / \sigma$ was estimated and is shown in Table III. $\Delta \sigma / \sigma$ increased as molecular weight decreased. Since we removed the proteins, phospholipids and fatty acids from natural rubber, change in $\Delta \sigma / \sigma$ may be due to unknown nucleating agents present in the rubber.

\section{Heterogeneous Nucleation of the Rubber}

To confirm the difference between the fatty acid and unknown nucleating agent, the transesterified NR (NRTE) mixed with $1 \mathrm{w} / \mathrm{w} \%$ stearic acid (NRTE-St) was used, in which NRTE was purified rubber before fractionation. Semi-logarithmic plots of $V$ vs. 1/T $\Delta T$ are shown in Figure 10. The slope and point of intersection of the line for NR were almost similar to those for NRTE and NRTE-St. This implies that, both $\sigma_{\mathrm{e}}$ and $\sigma$ are independent of molecular weight and fatty acids. Semi-logarithmic plots of $I$ vs. $1 / T \Delta T^{2}$ are shown in Figure 11. Logarithmic $I$ for NR was different from that for NRTE and NRTE-St. The slope of the line for NR was identical to the slope for NRTE-St, but different from the slope for NRTE.

$\Delta \sigma / \sigma$ for NR, NRTE and NRTE-St is shown in Table IV. $\Delta \sigma / \sigma$ for NRTE was about 5 times that for NR. Since $\Delta \sigma$ represents the ability of a nucleating agent, the difference in $\Delta \sigma / \sigma$ clearly indicates that fatty acids play the role of a nucleating agent. $\Delta \sigma / \sigma$

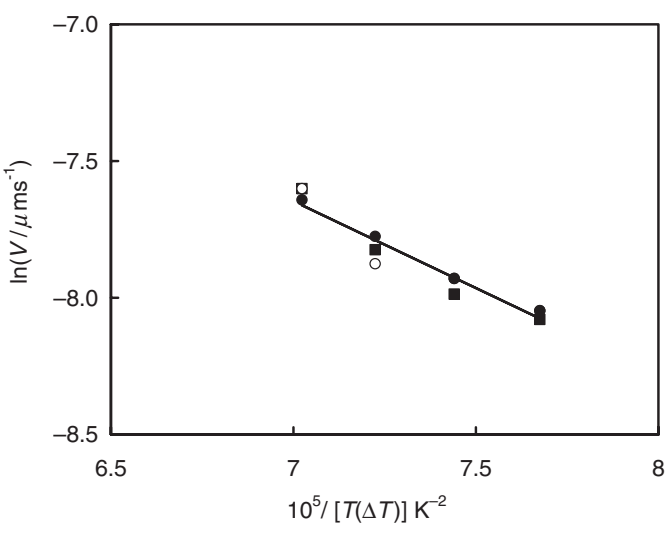

Figure 10. Semi-logarithmic plots of growth rate $v s .1 / T \Delta T$ for $(\boldsymbol{\Delta})$ natural rubber $(\mathrm{NR}),(\bigcirc)$ transesterified NR (NRTE), and (ם) NRTE mixed with $1 \mathrm{w} / \mathrm{w} \%$ stearic acid (NRTE-St).

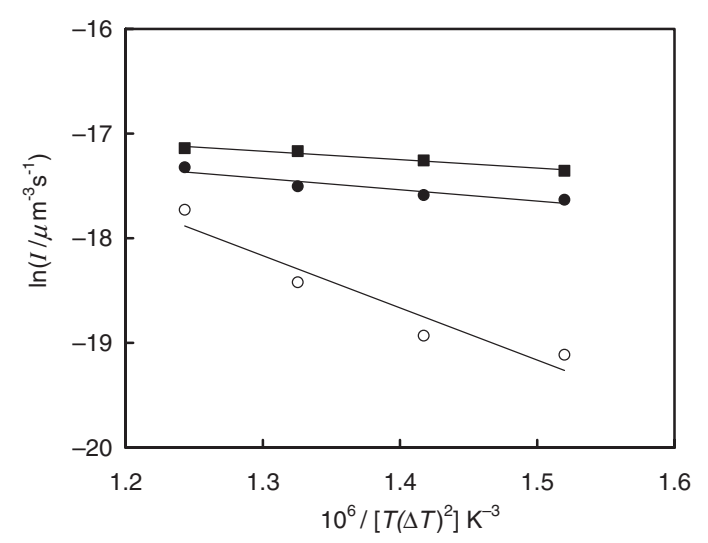

Figure 11. Semi-logarithmic plots of nucleation rate $v s$. $1 / T \Delta T^{2}$ for $(\mathbf{\Delta})$ natural rubber $(\mathrm{NR}),(\mathrm{O})$ transesterified NR (NRTE), and (ם) NRTE mixed with $1 \mathrm{w} / \mathrm{w} \%$ stearic acid (NRTE-St).

Table IV. $\Delta \sigma / \sigma$ for NR, NRTE and NRTE-St

\begin{tabular}{lc}
\hline Specimen & $\Delta \sigma / \sigma\left(10^{-2}\right)$ \\
\hline $\mathrm{NR}^{\mathrm{a}}$ & 2.1 \\
$\mathrm{NRTE}^{\mathrm{b}}$ & 9.7 \\
$\mathrm{NRTE}^{-} \mathrm{St}^{\mathrm{c}}$ & 1.6 \\
\hline \multicolumn{2}{c}{${ }^{\mathrm{a}}$ Natural rubber; ${ }^{\mathrm{b}}$ Transesterified } \\
natural rubber $\quad$ (NRTE); ${ }^{\mathrm{c}}$ Trans- \\
esterified natural rubber mixed with \\
$1 \mathrm{w} / \mathrm{w} \%$ stearic acid (NRTE-St).
\end{tabular}

for NRTE-St was 0.016 , similar to $\Delta \sigma / \sigma$ for NR and considerably less than for NRTE. Heterogeneous nucleation may thus be due to the effects of fatty acids. Comparing $\Delta \sigma / \sigma$ for NRTE and NRTE-St with those for fractionated rubbers, it is obvious that Fr1 contains more effective nucleating agent, while Fr4 less effective nucleating agent. Since the fractionated rubber was confirmed to contain less fatty acid, the effects of unknown nucleating agents on the rubber are suggested, even after transesterification. Stearic acid 
was found a better nucleating agent than unknown nucleating agents.

\section{CONCLUSIONS}

The crystallization of NR purified by deproteinization followed by transesterification was investigated by polarized light microscopy, in which spherulites were observed. The number and size of spherulites for purified NR increased linearly with crystallization time after induction. Based on these findings, we estimated nucleation rate, $I$, and growth rate, $V$, of crystals. These were inversely proportional to $T \Delta T^{2}$ and $T \Delta T$, respectively, as in the case of polyethylene. The side surface free energy $\sigma$, as well as fold surface free energy $\sigma_{\mathrm{e}}$, are thus independent of fatty acids. Only $\Delta \sigma$ depended on fatty acids and unknown nucleating agent. Fatty acids such as stearic acid were more effective as nucleating agents than unknown nucleating agents.

Acknowledgment. This work was supported in part by Grant-in-Aid for Science Research on Priority Areas, "Mechanism of Polymer Crystallization" (No. 12127205) and the 21st Century COE Program for Scientific Research from the Ministry of Education, Culture, Sports, Science and Technology, Japan.

\section{REFERENCES}

1. N. Bekkedahl, J. Res. Natl. Bur. Stad., 13, 411 (1934).

2. D. R. Burfield and Y. Tanaka, Polymer, 28, 907 (1987).

3. N. Nishiyama, S. Kawahara, T. Kakubo, A. H. Eng, and Y. Tanaka, Rubber Chem. Technol., 69, 608 (1996).
4. Y. Tanaka, S. Kawahara, and J. Tangpakdee, Kautsch. Gummi Kunst., 50, 6 (1997).

5. S. Kawahara, J. Sakdapipanich, Y. Isono, A. H. Eng, and Y. Tanaka, Rubber Chem. Technol., 75, 739 (2002).

6. P. J. Phillips and N. Vatansever, Macromolecules, 20, 2138 (1987).

7. A. H. Eng, S. Ejiri, S. Kawahara, and Y. Tanaka, J. Appl. Polym. Sci., Appl. Polym. Symp., 53, 5 (1994).

8. A. H. Eng, S. Kawahara, and Y. Tanaka, J. Nat. Rubber Res., 8, 109 (1993).

9. J. Tangpakdee and Y. Tanaka, J. Rubber Res., 1, 14 (1998).

10. J. Tangpakdee and Y. Tanaka, J. Nat. Rubber Res., 12, 112 (1997).

11. A. H. Eng, S. Kawahara, and Y. Tanaka, Rubber Chem. Technol., 67, 159 (1994).

12. A. Subramanian, Rubber Res. Inst. Malays. Technol. Bull., 4, 1 (1980).

13. E. H. Andrews, P. J. Owen, and A. Singh, Proc. R. Soc. London, Ser. A, 324, 79 (1971).

14. S. Kawahara, Y. Inomata, T. Kakubo, Y. Tanaka, K. Hatada, K. Ute, and N. Miyatake, Rubber Chem. Technol., 71, 277 (1998).

15. E. N. Dalal, K. D. Taylor, and P. J. Phillips, Polymer, 24, 1623 (1983).

16. H. G. Kim and L. Manderkern, J. Polym. Sci., Part A-2: Polym. Phys., 10, 1125 (1972).

17. S. Kawahara, K. Takano, Y. Isono, and M. Hikosaka, J. Macromol. Sci. Phys., B, 42, 569 (2003).

18. F. P. Price, "Nucleation in Polymer Crystallization," Nucleation, Marcel Dekker, Inc., New York, N.Y., 1969, Chapter 8.

19. E. N. Dalal and P. J. Phillips, J. Polym. Sci., Polym. Lett. Ed., 22, 7 (1984).

20. M. Nishi, M. Hikosaka, S. K. Ghosh, A. Toda, and K. Yamada, Polym. J., 31, 749 (1999). 